\title{
Eltrombopag: an update on the novel, non-peptide thrombopoietin receptor agonist for the treatment of immune thrombocytopenia
}

\author{
Thomas Kühne • Paul Imbach
}

Received: 17 November 2009 / Accepted: 22 March 2010/Published online: 20 April 2010

(C) Springer-Verlag 2010

\begin{abstract}
Immune thrombocytopenia (ITP) is characterised by a transient or persistent decrease in platelets accompanied by an increased risk of bleeding, which can have a significant negative impact on patients' health-related quality of life. The condition has long been associated with an increased rate of immune-mediated platelet destruction, and traditional treatments have targeted the reduction in platelet destruction; however, some interventional drugs are limited by transient efficacy and side effects. Recent advances in our understanding of ITP pathogenesis have highlighted the role of impaired platelet production, which has led to the advent of a new generation of thrombopoietin (TPO)-receptor agonist therapies, including eltrombopag and romiplostim. The oral, non-peptide TPO-receptor agonist eltrombopag has shown considerable promise in both preclinical and clinical trials. Eltrombopag has a unique mechanism of action and binds to a transmembrane region of the TPO receptor that is distant from the TPO binding site. As such, eltrombopag may confer synergistic effects with endogenous TPO rather than competing for binding. Eltrombopag also induces activation of the TPO receptor and downstream signalling in a distinct manner to TPO and does not have a significant impact on platelet function. Clinical evidence demonstrates that eltrombopag produces a rapid and sustainable increase in platelet counts that significantly reduces bleeding and is well tolerated in patients with ITP. Eltrombopag therefore represents an important addition to the therapeutic armamentarium for ITP.
\end{abstract}

T. Kühne $(\bowtie) \cdot$ P. Imbach

Pediatric Hematology-Oncology, University Children's Hospital,

Medical Faculty of University of Basel UKBB,

PO Box, CH-4005 Basel, Switzerland

e-mail: thomas.kuehne@ukbb.ch
Keywords Thrombocytopenia $\cdot$ ITP. Thrombopoietin . TPO $\cdot$ Eltrombopag

\section{Introduction}

Immune thrombocytopenia (ITP; formerly idiopathic or immune thrombocytopenic purpura) is mostly an acquired cytopenia of adults and children with various aetiologies. It is characterised by a transient or persistent decrease in platelets and an increase in the risk of bleeding [1-5]. The estimated incidence of ITP is 100 cases per 1 million persons per year, with approximately $50 \%$ of these cases occurring in children [2, 6]. In adults, the incidence is higher in women than in men [2], whereas the reverse is true in children, where ITP predominates in young male [7]. Clinical presentation varies from asymptomatic or mild symptoms (bruising and petechiae) to mucocutaneous bleeding that can become severe $[5,8]$.

ITP has long been known to be associated with an increased rate of immune-mediated platelet destruction. The exact mechanism of this destruction remains unknown, and there may be several immune-mediated co-mechanisms [3]. Traditional treatment strategies for patients with ITP have focused on reducing platelet destruction. However, with greater insight into the pathophysiology of ITP, it has become apparent that this disorder results not only from increased platelet destruction but also from impaired platelet production [9]. These processes are thought to result from auto-antibodies targeting both mature platelets and megakaryocytes for destruction by macrophages [9]. These new findings have driven the concept of targeting platelet stimulation in the treatment of ITP and the subsequent introduction of a new generation of thrombopoietin (TPO)receptor agonists, including eltrombopag and romiplostim. 


\section{The role of thrombopoietin in platelet production}

Although some platelets are consumed in the haemostatic process, platelets typically remain in circulation for approximately 10 days before destruction [10]. In order to achieve a stable platelet count, platelet production must equal the rate of platelet consumption and destruction. Platelet production, or thrombopoiesis, occurs in the bone marrow where committed stem cells differentiate into megakaryocytes. Platelets are formed from haematopoietic stem cells through a series of divisions of progenitor cells, which ultimately produce the mature megakaryocytes responsible for platelet release (Fig. 1) [11].

The glycoprotein hormone TPO, produced primarily in the liver, is the principal regulator of megakaryocyte development and platelet production (Fig. 1) [11-13].

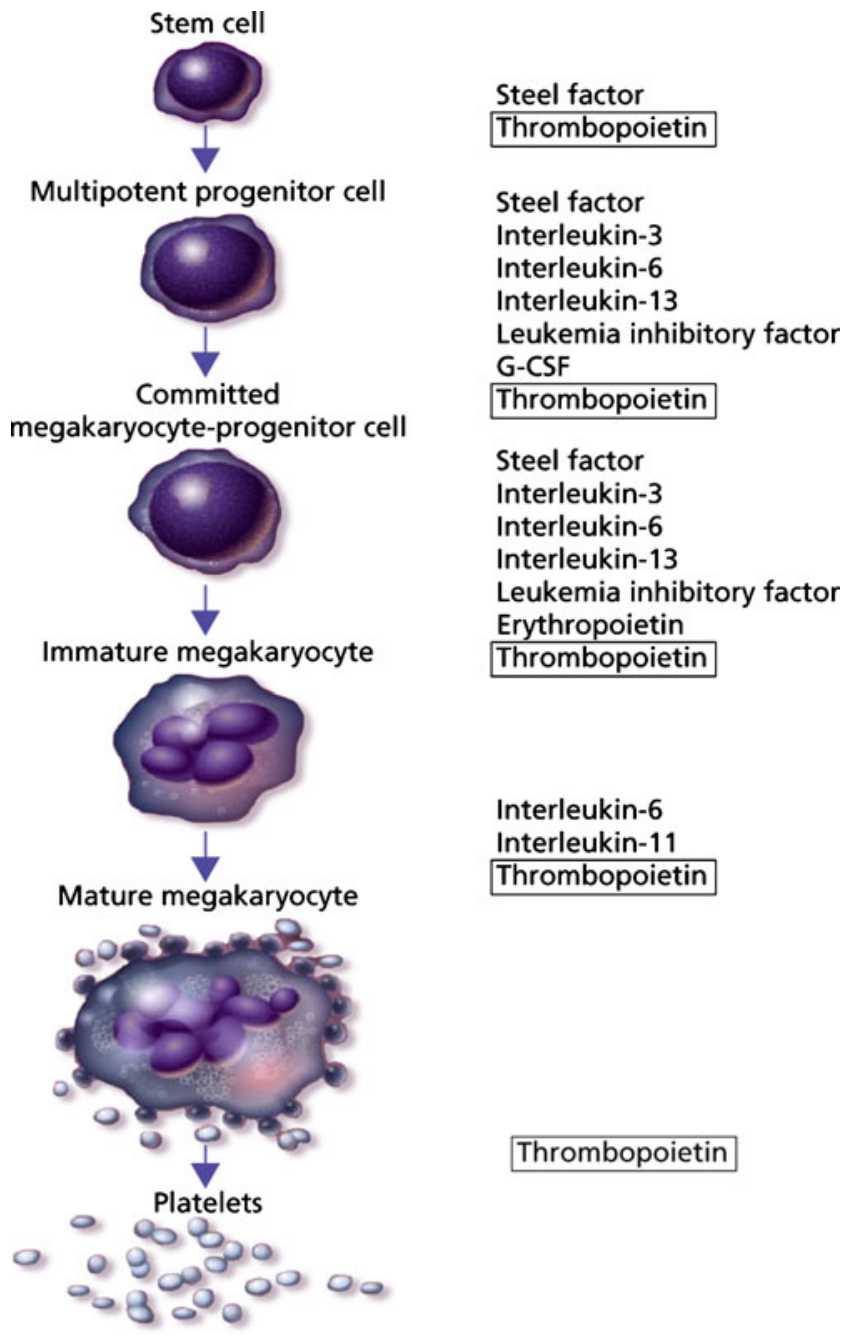

Fig. 1 The role of cytokines in megakaryocytopoiesis. Many stages of megakaryocyte development can be affected by several cytokines in vitro, including steel factor (also termed stem-cell factor, mast-cell growth factor, or Kit ligand), interleukin-3, interleukin-6, interleukin11, granulocyte colony-stimulating factor (G-CSF), erythropoietin, leukaemia inhibitory factor and thrombopoietin [11]
Thrombopoietin is involved in nearly every stage of megakaryocyte development, supporting cell survival and cell cycling, as well as modulating apoptosis and cell cycle regulators [14]. Thrombopoietin acts by binding and activating cell surface TPO receptors (also known as cMpl) [11, 15]. The activated TPO receptor undergoes dimerisation and a conformation change that results in activation of Janus protein tyrosine kinases (JAK) and subsequent downstream signalling within the megakaryocyte via signal transducers and activators of transcription (STAT), phosphoinositide 3-kinase (PI3K), and mitogenactivated protein kinases (MAPK) effectors. This induces megakaryocyte proliferation and differentiation, which leads to increased platelet production $[12,16]$.

In individuals without ITP, a drop in platelet levels triggers an increase in TPO in order to stimulate platelet production and correct the platelet deficit [14, 15, 17-20]. However, measurement of TPO in patients with ITP has repeatedly shown levels to be within the normal range or only slightly elevated, and there does not appear to be a correlation between platelet counts and serum TPO levels in ITP patients $[17,18,20]$. Thus, the new class of TPOreceptor agonists aims to increase platelet production by supplementing the suboptimal levels of endogenous TPO present in many individuals with ITP.

\section{Treatment for immune thrombocytopenia}

The principal aim of treatment for ITP is to achieve a platelet count that will provide protection against the risk of major bleeding rather than to correct patients' platelet counts to normal levels [21]. Current treatment strategies are hampered by the heterogeneous nature of ITP with regard to its severity and clinical course and its unpredictable response to therapy. Standard treatment options include corticosteroids, intravenous immunoglobulins, anti-D-immunoglobulins and surgical splenectomy; however, all are limited by their often transient efficacy [2] and side effects, which can be difficult to tolerate [22]. Splenectomised patients have an increased long-term risk of infection. Splenectomy is rarely performed in children given both the increased likelihood of spontaneous and/or therapyinduced remission and the increased infection-related morbidity and mortality [23]. Rituximab, a monoclonal anti-CD20 antibody, is an effective immunotherapeutic agent increasingly used to treat refractory ITP; however, its use is associated with infusion-related side effects [24, 25], increased risk of the development of progressive multifocal leukoencephalopathy [26] and a greater incidence of infections [27].

Current treatments primarily target platelet destruction $[2,28]$. As such, the development of new therapies has focused on stimulation of platelet production, including 
growth factor and growth factor analogue stimulation of megakaryopoiesis [4, 29, 30]. Although the first generation agents demonstrated efficacy in elevating platelet counts, some of them were associated with the development of autoantibodies that cross-react with, and neutralise, endogenous TPO, leading to severe thrombocytopenia [30]. Therefore, research into other agents, including non-peptide TPOreceptor agonists that do not trigger immunogenicity, was initiated [30].

In 2008, two new drugs, romiplostim $\left(\right.$ Nplate $\left.^{\mathrm{TM}}\right)$ and eltrombopag (Revolade $^{\mathrm{TM}}$, known as Promacta ${ }^{\circledR}$ in the USA and currently under European Medicines Agency review) that mimic the effect of TPO became available to treat adults with chronic ITP [31]. Romiplostim is a peptide mimetic given by subcutaneous injection that activates the TPO receptor via a similar mechanism to TPO, resulting in increases in platelet count in healthy humans as well as in over $80 \%$ of patients with ITP [31]. Eltrombopag is a novel oral, small molecule (molecular weight 442.5 Da for the free acid), synthetic non-peptide TPO-receptor agonist that has shown considerable promise in preclinical and clinical trials, with increases in platelet counts to $\geq 50,000 / \mu \mathrm{L}$ reported in $59-87 \%$ of patients with ITP [32-35].

\section{Eltrombopag}

Eltrombopag displays receptor-specific and species-specific binding

In preclinical studies, eltrombopag has been shown to stimulate human megakaryocyte differentiation and proliferation in a dose-dependent manner, via stimulation of the TPO receptor [36].

Eltrombopag displays high receptor- and species-specific binding selectivity for the human TPO receptor. The response of normal human TPO receptors has been elucidated by measuring growth and differentiation of human bone marrow in vitro. These studies demonstrated human and chimpanzee-specific stimulation of TPOreceptor-expressing cells via activation of STAT5, with no eltrombopag-induced STAT activation observed in other species tested, including cynomolgus macaques, rhesus monkeys, pigs, dogs, ferrets, rabbits, rats and mice [36]. The selectivity of eltrombopag for the TPO-receptor rather than other growth factor receptors was demonstrated by assays measuring STAT activation or reporter gene expression in various transfected and non-transfected cell lines. The assays confirmed the ability of eltrombopag to activate signalling in cells expressing the TPO receptor but showed that it had no effect on cells expressing only interferon- $\gamma$, granulocyte macrophage-colony stimulating factor (GMCSF), G-CSF, erythropoietin or interleukin-3 receptors
[36]. The fact that eltrombopag lacks activity against cells expressing growth factor receptors other than TPO, even those acting through the JAK/STAT signalling pathway, suggests that the activation of JAK/STAT by eltrombopag is due to the specific activation of the TPO receptor [37].

Additional studies have demonstrated that eltrombopag specificity can also be observed at a cellular level. In ex vivo experiments, eltrombopag effectively increased megakaryocytic colony formation from mature and immature megakaryocyte progenitors [38]. However, treatment with eltrombopag did not result in a significant change in differentiation nor maturation characteristics of the granulocytic/monocytic or the erythrocytic lineages, indicating that eltrombopag preferentially acts on progenitor and precursor cells harbouring megakaryocytic potential [38]. Thus, eltrombopag is a true agonist of the human TPO receptor that is able to act on megakaryocytes and their progenitors in a manner similar to TPO with the potential to induce differentiation and proliferation of megakaryocytes and ultimately relieve thrombocytopenia [37].

\section{Eltrombopag: unique mechanism of action}

While both eltrombopag and romiplostim act as TPO agonists, their mechanisms of action differ, since they bind and activate the TPO receptor in different ways [39]. Romiplostim binds directly to the receptor in the same manner as endogenous TPO and initiates downstream signalling in a similar manner to endogenous TPO [39]. In vitro studies have demonstrated that romiplostim competes with endogenous TPO for binding [40]. In contrast, eltrombopag interacts with the TPO receptor at a binding site distant from that of TPO and appears to initiate signal transduction by a mechanism different from that of endogenous TPO [39] (Fig. 2). Since eltrombopag and TPO do not bind to the same site, competitive binding is avoided, and eltrombopag and TPO confer additive cellsignalling effects [37] (Fig. 2).

The ultimate responses to eltrombopag are similar to those observed with endogenous TPO with stimulation of transcription through STAT-based and megakaryocytespecific promoters [37]. However, studies have reported some differences in activation of downstream targets of the TPO receptor between eltrombopag and human recombinant TPO (hrTPO). In vitro studies have shown that while treatment with hrTPO leads to a significant increase of both STAT3 and STAT5 phosphorylation, cells treated with eltrombopag respond with an increase in STAT5 phosphorylation only, while p-STAT3 levels remain consistent with levels observed in untreated controls [38]. In their signal transduction studies, Erhardt et al. reported that eltrombopag induced the phosphorylation of STAT proteins, but not of Akt, whereas hrTPO induced the phosphorylation of Akt 
Fig. 2 Cellular mechanism of action of eltrombopag. Eltromdomain of the TPO-receptor distal from the TPO binding site to initiate TPO signalling by a distinct mechanism that leads to increased platelet production bopag binds to a transmembrane

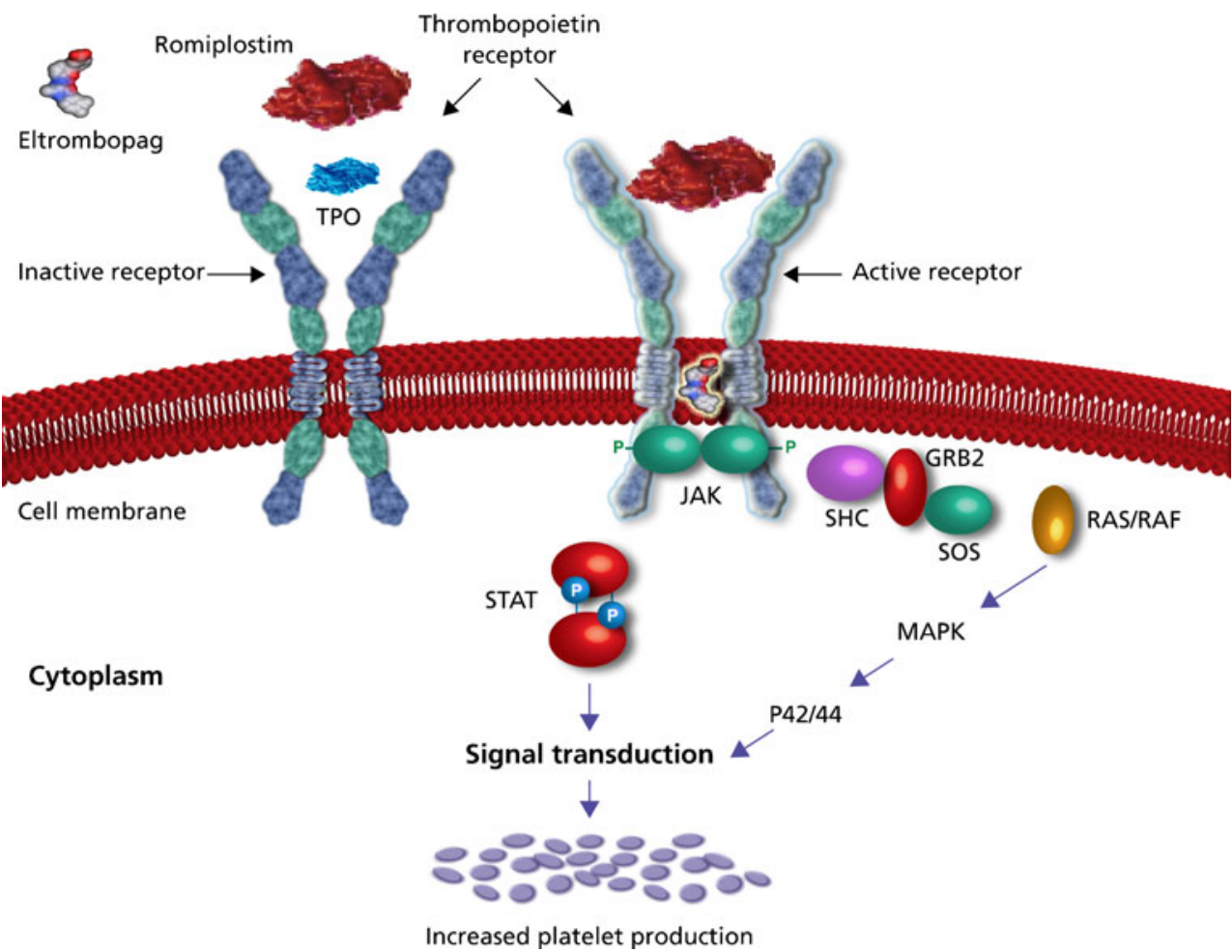

Efficacy

in addition to the STAT proteins [41]. These findings suggest that the effect of eltrombopag appears to be distinct from hrTPO or other TPO mimetics in some respects and may be explained by eltrombopag's unique binding site on the TPO receptor.

TPO and eltrombopag may also differ with regard to their effects on platelet activation. In vitro, TPO primes platelets to aggregate in the presence of other substances, such as adenosine diphosphate or collagen, which stimulate platelet aggregation [42]. Although it is unclear whether platelet 'priming' represents any risk for patients, it may pose a risk in patients prone to thrombogenic conditions [11]. Eltrombopag stimulates platelet production with little or no effect on platelet function. In vitro and ex vivo studies using platelet samples from healthy volunteers and patients with chronic ITP have shown that eltrombopag has no effect on platelet aggregation or activation [41, 43] and that platelet function with eltrombopag is comparable to that with placebo [43]. The clinical relevance of these findings is unknown at present.

\section{Clinical experience with eltrombopag}

Consistent with its proposed mechanism of action, eltrombopag has demonstrated efficacy with a well-tolerated safety profile in an extensive clinical trial program of over 400 patients with chronic ITP in phase II and III studies (Table 1).
In adults with chronic ITP, eltrombopag therapy $(50 \mathrm{mg} /$ day once daily) has been shown to rapidly increase platelet counts to haemostatic levels $\left(>50 \times 10^{9} / \mathrm{L}\right)$ within 15 days, with over $50 \%$ of patients achieving platelet counts $\geq 50 \times 10^{9} / \mathrm{L}$ on day 43 (Fig. 3) [34]. Responses are achieved regardless of baseline platelet levels, concomitant ITP therapy or splenectomy status [33, 34]. In the long-term Randomised Placebo-Controlled ITP Study with Eltrombopag (RAISE), from day 15 throughout treatment, median platelet counts were between 52 and $91 \times 10^{9} / \mathrm{L}$ for patients treated with eltrombopag; in contrast, median platelet counts for the placebo group never exceeded $30 \times 10^{9} / \mathrm{L}$ [44].

Eltrombopag therapy was also associated with a significant reduction in the incidence of World Health Organisation (WHO)-defined bleeding [45]. In the 6-month RAISE, treatment with eltrombopag was associated with a $76 \%$ reduction in the odds of bleeding (WHO grades 1-4) over 6 months compared with placebo [OR, 0.24 (95\% CI, $0.16-$ $0.38) ; p<0.001]$ and a $65 \%$ reduction in the odds of clinically significant bleeding (WHO grades 2-4) over 6 months compared with placebo [OR, 0.35 (95\% CI, 0.190.64); $p<0.001$ ) [45].

Eltrombopag therapy may also allow ITP patients to discontinue concomitant medications and reduce their rescue medication usage. In the 6-month RAISE, more patients receiving eltrombopag stopped or dose-reduced 


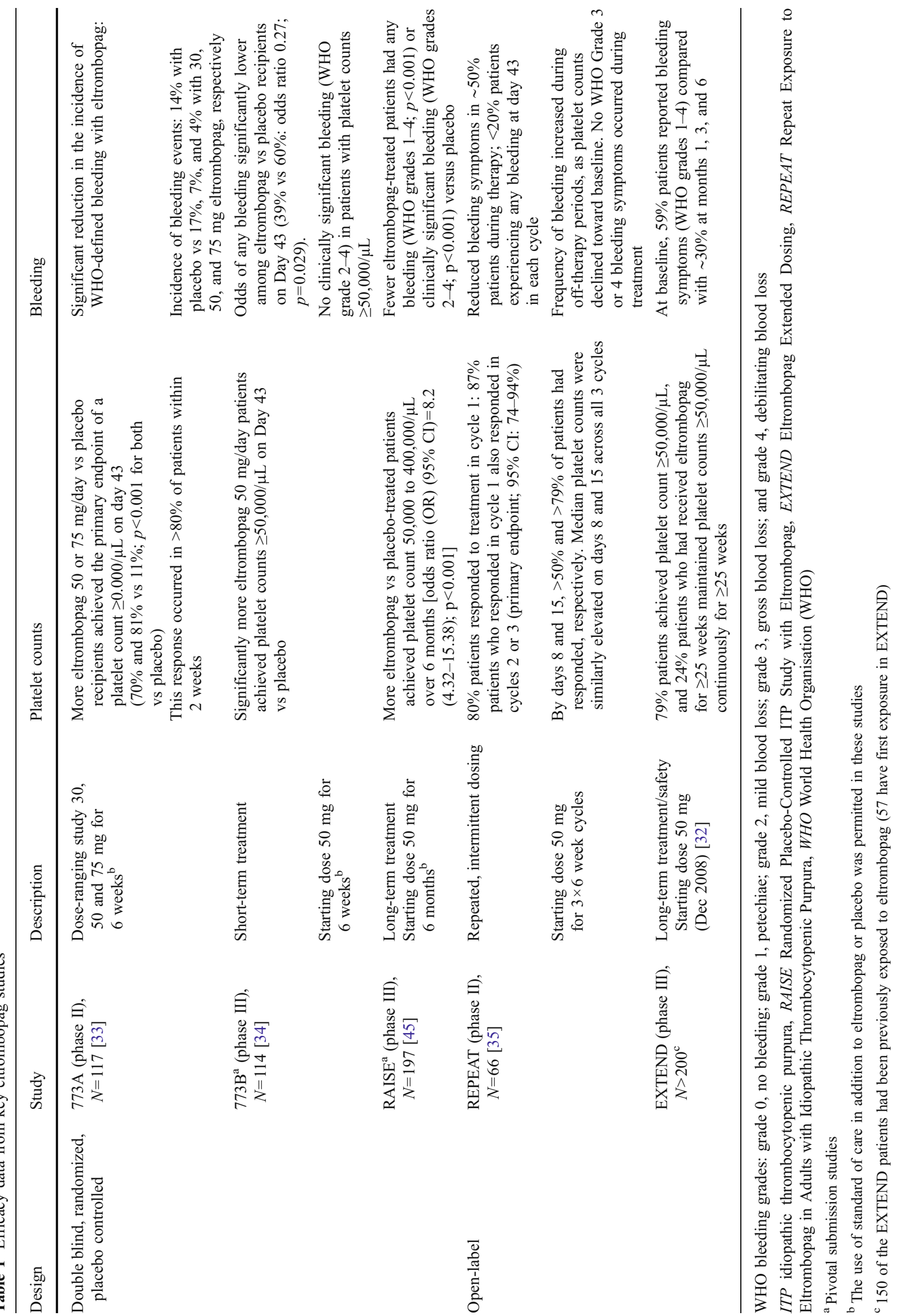




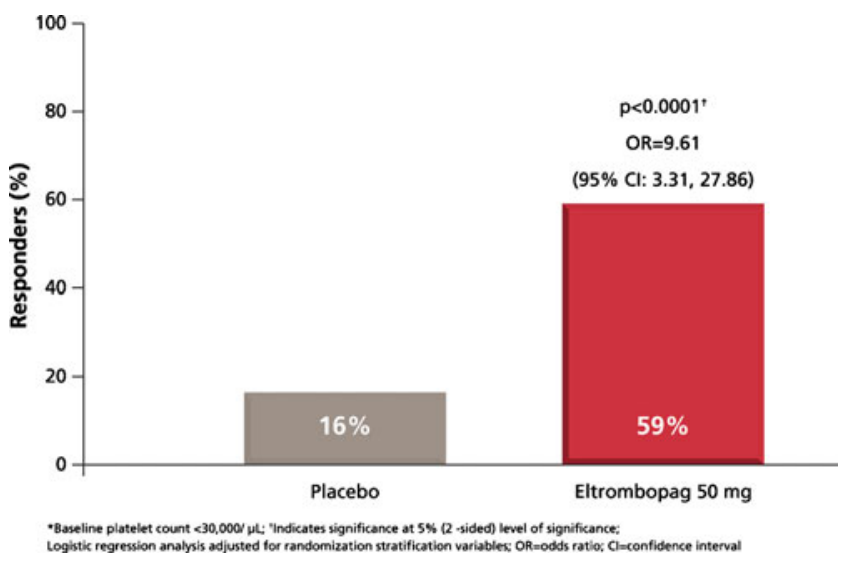

Fig. 3 Proportion of patients who achieved a platelet count of $\geq 50,000 / \mu \mathrm{L}$ on day 43 following a 6 -week treatment period in a randomized, double-blind, placebo-controlled study of eltrombopag in patients with chronic ITP [34]

their concomitant ITP medications versus placebo (59 vs $32 \% ; p=0.016$ ), and significantly fewer patients required rescue therapy with eltrombopag versus placebo (19 vs $40 \% ; p=0.001)$ [45]. In the Eltrombopag Extended Dosing (EXTEND) study, $70 \%$ of patients who responded to eltrombopag were able to discontinue their concomitant ITP medication or had a sustained reduction in their baseline ITP medication and did not require subsequent rescue medication [46].

The efficacy of eltrombopag against the symptoms of ITP translated into improvements in health-related quality of life measures. Results from the 6-month RAISE study showed that patients receiving eltrombopag reported meaningful improvements in vitality, emotional and physical role domains and in overall mental health [47]. Eltrombopagtreated patients consistently reported clinically and statistically significant benefits in physical and social activities and concerns around bleeding and bruising symptoms. They also perceived a reduction in fatigue symptoms and an improved ability to participate in activities of daily living $[47,48]$.

\section{Safety}

Eltrombopag was generally well tolerated in clinical trials, with an adverse event (AE) profile that was comparable to that of placebo. In the phase II and III trials, the incidence and severity of AEs with eltrombopag were similar to those reported with placebo, with most events being mild to moderate in severity. Headache was reported to be the most common AE (incidence, 8-30\%) [33, 34, 45]. In RAISE, a higher incidence of hepatobiliary laboratory abnormalities were reported in the eltrombopag- (13\%) versus placebotreated patients (7\%) [45], and the gastrointestinal system was the most commonly affected system [45]. The most common serious adverse event was haemorrhage; most haemorrhagic events occurred when platelet counts were decreasing after cessation of eltrombopag therapy [33, 34, 45]. Although transient decreases in platelet counts below baseline levels were reported in several eltrombopag-treated patients in the phase III trial, these were not accompanied by clinically meaningful increases in bleeding [34]. In the long-term EXTEND study, where the median time on eltrombopag treatment was 91.5 days (range, 2-523 days), seven patients experienced thromboembolic events; four of these events were considered to be related to study medication [32]. Five patients had reticulin, and two patients had reticulin in conjunction with collagen fibres documented in the bone marrow; three of these patients had documentation of pre-existing reticulin fibres [32].

Eltrombopag was also well tolerated upon repeated administration in the Repeat Exposure to Eltrombopag in Adults with Idiopathic Thrombocytopenic Purpura (REPEAT) study [35] and as long-term therapy in the 6month RAISE and the ongoing open-label phase III extension EXTEND studies, in which the safety profiles were similar to those reported in the 6-week phase III studies [32, 34, 45, 49].

\section{Conclusions}

Although often considered a benign condition, ITP has a significant negative impact on patients' health-related quality of life, and this impact is often exacerbated by the adverse effects of traditional treatments such as corticosteroids [34]. Recent advances in our understanding of chronic ITP have highlighted the key role of impaired platelet production in the pathogenesis of the condition, and this in turn has led to the advent of a new class of TPOreceptor agonist therapies.

The oral, non-peptide TPO-receptor agonist eltrombopag has shown considerable promise in both preclinical and clinical trials. Eltrombopag has a unique mechanism of action, binding to a transmembrane region of the TPO receptor that is distant from the TPO binding site, meaning that it may confer synergistic effects with endogenous TPO rather than competing for binding. It induces activation of the TPO receptor and downstream signalling in a distinct manner to TPO and does not have a significant impact on platelet function. Clinical evidence demonstrates that eltrombopag is well tolerated in patients with ITP and produces a rapid and sustainable increase in platelet counts, which significantly reduces bleeding. Thus, eltrombopag represents an important addition to the therapeutic armamentarium for ITP and a step toward shifting the treatment paradigm of ITP to include platelet production in addition to preventing platelet destruction. 
Acknowledgments The editors of this supplement invited GlaxoSmithKline and Amgen as two of the sponsors of the Intercontinental Cooperative ITP Study (ICIS) group expert meeting (see editorial) to update the group on their new TPO agonists. GlaxoSmithKline asked TK and PI to author this article based on the discussions at the ICIS meeting. The content of the article was reviewed for scientific accuracy by Dr. Luisa Veronese and Richard Brun of GlaxoSmithKline. Editorial support was provided by Huw Jones of Medicus International and funded by GlaxoSmithKline.

\section{References}

1. Chang M, Nakagawa P, Williams S et al (2003) Immune thrombocytopenic purpura (ITP) plasma and purified ITP monoclonal autoantibodies inhibit megakaryocytopoiesis in vitro. Blood 102:887-895

2. Cines DB, Blanchette VS (2002) Immune thrombocytopenic purpura. N Engl J Med 346:995-1008

3. Cooper N, Bussel J (2006) The pathogenesis of immune thrombocytopaenic purpura. Br J Haematol 133:364-374

4. Rodeghiero F, Michel M, Provan D et al (2008) The burden of chronic idiopathic thrombocytopenic purpura (ITP) in adults: results of a chart review of 610 patients in Europe. Paper presented at the EHA 13th Congress, Copenhagen, 12-15 June 2008

5. Stasi R, Provan D (2004) Management of immune thrombocytopenic purpura in adults. Mayo Clin Proc 79:504-522

6. Neylon AJ, Saunders PW, Howard MR, Proctor SJ, Taylor PR (2003) Clinically significant newly presenting autoimmune thrombocytopenic purpura in adults: a prospective study of a population-based cohort of 245 patients. Br J Haematol 122:966974

7. Kuhne T, Imbach P, Bolton-Maggs PH, Berchtold W, Blanchette V, Buchanan GR (2001) Newly diagnosed idiopathic thrombocytopenic purpura in childhood: an observational study. Lancet 358:2122-2125

8. Cines D, Bussel J (2005) How I treat idiopathic thrombocytopenic purpura (ITP). Blood 106:2244-2251

9. Gernsheimer T (2008) Epidemiology and pathophysiology of immune thrombocytopenic purpura. Eur J Haematol Suppl (69):38

10. Leeksma CH, Cohen JA (1955) Determination of the life of human blood platelets using labelled diisopropylfluorophosphanate. Nature 175:552-553

11. Kaushansky K (1998) Thrombopoietin. N Engl J Med 339:746754

12. Kaushansky K (2005) The molecular mechanisms that control thrombopoiesis. J Clin Invest 115:3339-3347

13. Kuter DJ, Begley CG (2002) Recombinant human thrombopoietin: basic biology and evaluation of clinical studies. Blood 100:3457-3469

14. Gernsheimer T (2009) Chronic idiopathic thrombocytopenic purpura: mechanisms of pathogenesis. Oncologist 14:12-21

15. von dem Borne A, Folman C, van den Oudenrijn S, Linthorst G, de Jong S, de Haas M (2002) The potential role of thrombopoietin in idiopathic thrombocytopenic purpura. Blood Rev 16:57-59

16. Stasi R, Evangelista ML, Amadori S (2008) Novel thrombopoietic agents: a review of their use in idiopathic thrombocytopenic purpura. Drugs 68:901-912

17. Mukai HY, Kojima H, Todokoro K et al (1996) Serum thrombopoietin (TPO) levels in patients with amegakaryocytic thrombocytopenia are much higher than those with immune thrombocytopenic purpura. Thromb Haemost 76:675-678
18. Aledort LM, Hayward CP, Chen MG, Nichol JL, Bussel J (2004) Prospective screening of 205 patients with ITP, including diagnosis, serological markers, and the relationship between platelet counts, endogenous thrombopoietin, and circulating antithrombopoietin antibodies. Am J Hematol 76:205-213

19. Emmons RV, Reid DM, Cohen RL et al (1996) Human thrombopoietin levels are high when thrombocytopenia is due to megakaryocyte deficiency and low when due to increased platelet destruction. Blood 87:4068-4071

20. Kosugi S, Kurata Y, Tomiyama Y et al (1996) Circulating thrombopoietin level in chronic immune thrombocytopenic purpura. Br J Haematol 93:704-706

21. Rodeghiero F, Stasi R, Gernsheimer T et al (2009) Standardization of terminology, definitions and outcome criteria in immune thrombocytopenic purpura of adults and children: report from an international working group. Blood 113:2386-2393

22. George JN (2006) Management of patients with refractory immune thrombocytopenic purpura. J Thromb Haemost 4:1664 1672

23. Bisharat N, Omari H, Lavi I, Raz R (2001) Risk of infection and death among post-splenectomy patients. J Infect 43:182-186

24. Arnold DM, Dentali F, Crowther MA et al (2007) Systematic review: efficacy and safety of rituximab for adults with idiopathic thrombocytopenic purpura. Ann Intern Med 146:25-33

25. Zhou Z, Yang R (2008) Rituximab treatment for chronic refractory idiopathic thrombocytopenic purpura. Crit Rev Oncol Hematol 65:21-31

26. Carson KR, Evens AM, Richey EA et al (2009) Progressive multifocal leukoencephalopathy after rituximab therapy in HIVnegative patients: a report of 57 cases from the Research on Adverse Drug Events and Reports project. Blood 113:4834-4840

27. Arnold DM, Nazi I, Kelton JG (2009) New treatments for idiopathic thrombocytopenic purpura: rethinking old hypotheses. Expert Opin Investig Drugs 18:805-819

28. Bussel J (2006) Treatment of immune thrombocytopenic purpura in adults. Semin Hematol 43:S3-S10, discussion S18-S19

29. Psaila B, Bussel J, vasey S et al (2008) Efficacy and safety of repeated intermittent treatment with eltrombopag in patients with chronic idiopathic thrombocytopenic purpura. Paper presented at the EHA 13th Congress, Copenhagen, 12-15 June 2008

30. Newland A (2007) Thrombopoietin mimetic agents in the management of immune thrombocytopenic purpura. Semin Hematol 44:S35-S45

31. Kuter DJ (2009) Thrombopoietin and thrombopoietin mimetics in the treatment of thrombocytopenia. Annu Rev Med 60:193-206

32. Bussel J, Cheng G, Saleh M et al (2008) Safety and efficacy of long-term treatment with oral eltrombopag for chronic idiopathic thrombocytopenic purpura. Presented at the American Society of Hematology 50th Annual Meeting, San Francisco, 6-9 December 2008

33. Bussel JB, Cheng G, Saleh MN et al (2007) Eltrombopag for the treatment of chronic idiopathic thrombocytopenic purpura. N Engl J Med 357:2237-2247

34. Bussel JB, Provan D, Shamsi T et al (2009) Effect of eltrombopag on platelet counts and bleeding during treatment of chronic idiopathic thrombocytopenic purpura: a randomised, double-blind, placebo-controlled trial. Lancet 373:641-648

35. Bussel JB, Psaila B, Saleh MN et al (2008) Efficacy and safety of repeated intermittent treatment with eltrombopag in patients with chronic idiopathic thrombocytopenic purpura. Blood 112:3431

36. Erickson-Miller CL, DeLorme E, Tian SS et al (2005) Discovery and characterization of a selective, nonpeptidyl thrombopoietin receptor agonist. Exp Hematol 33:85-93

37. Erickson-Miller CL, Delorme E, Tian SS et al (2009) Preclinical activity of eltrombopag (SB-497115), an oral, nonpeptide thrombopoietin receptor agonist. Stem Cells 27:424-430 
38. Will B, Kawahara M, Luciano JP et al (2009) Effect of the nonpeptide thrombopoietin receptor agonist eltrombopag on bone marrow cells from patients with acute myeloid leukemia and myelodysplastic syndrome. Blood 114:3899-3908

39. Evangelista M, Amadori S, Stasi R (2007) Biologic aspects of thrombopoietin and the development of novel thrombopoietic agents for clinical use. Curr Drug Discov Technol 4:162-173

40. Broudy V, Lin N (2004) AMG 531 stimulates megakaryopoiesis in vitro by binding to Mpl. Cytokine 25:52-60

41. Erhardt JA, Erickson-Miller CL, Aivado M, Abboud M, Pillarisetti K, Toomey JR (2009) Comparative analyses of the small molecule thrombopoietin receptor agonist eltrombopag and thrombopoietin on in vitro platelet function. Exp Hematol 37:1030-1037

42. Harker LA, Hunt P, Marzec UM et al (1996) Regulation of platelet production and function by megakaryocyte growth and development factor in nonhuman primates. Blood 87:1833-1844

43. Jenkins JM, Williams D, Deng Y et al (2007) Phase 1 clinical study of eltrombopag, an oral, nonpeptide thrombopoietin receptor agonist. Blood 109:4739-4741

44. Cheng G, Saleh MN, Bussel JB et al (2008) Oral eltrombopag for the long-term treatment of patients with chronic idiopathic thrombocytopenic purpura: results of a phase III, double- blind, placebo-controlled study (RAISE). Blood 112:Abstract 400

45. Cheng G, Saleh M, Bussel J et al (2008) Oral Eltrombopag for the long-term treatment of patients with chronic idiopathic thrombocytopenic purpura: results of a phase III, double-blind, placebo-controlled study (RAISE). Presented at the American Society of Hematology 50th Annual Meeting, San Francisco, 6-9 December 2008

46. Fogarty P, Bussel J, Cheng G et al (2008) Oral eltrombopag reduces the need for concomittant medications in patients with chronic idiopathic thrombocytopenic purpura. Presented at the American Society of Hematology 50th Annual Meeting, San Francisco, 6-9 December 2008

47. Provan D, Bussel JB, Cheng G et al (2009) Improvement in fatigue and health-related quality of life (HRQoL) with long-term eltrombopag therapy in chronic idiopathic thrombocytopenic purpura: results of phase 3, double-blind study (RAISE). Haematologica 94:Abstract 233

48. McMillan R, Bussel JB, George JN, Lalla D, Nichol JL (2008) Self-reported health-related quality of life in adults with chronic immune thrombocytopenic purpura. Am J Hematol 83:150-154

49. (2009) Revolade (eltrombopag) European summary of product characteristics (draft submission 3; not yet approved) 